\title{
Thermal-electrical analogy and inertia for thermal performance of building envelops
}

\author{
Rafik Absi $^{1, *}$, Stéphane Marchandon ${ }^{2}$, Rachid Bennacer $^{3}$ \\ ${ }^{1}$ Quartz (EA 7393), LR2E-Lab, ECAM-EPMI, 13, Boulevard de 1'Hautil, 95092, Cergy-Pontoise, France \\ ${ }^{2}$ EBI, EBInnov-Lab, 49 Avenue des Genottes CS90009 95895, Cergy-Pontoise, France \\ ${ }^{3}$ LMT, ENS Paris-Saclay, CNRS, Université Paris-Saclay, 94235 Cachan, France
}

\begin{abstract}
For transient thermal performance of building envelops adequate parameters are needed to capture the time lag and decrement factor. It is surprising that, in the formal electrical analogy, "inertia" is not represented by same components in fluid mechanics and heat transfer. In Windkessel model for fluid flow in elastic tubes, the fluid inertia is represented by an electrical inductance while in thermal-electric analogy, thermal inertia is given by a capacitance. Some authors argued that the terminology of "thermal inertia" is used incorrectly in the literature. The aim of our communication is to provide some clarification about this controversy. We will show that the thermal effusivity which is the geometric mean of thermal conductivity and volumetric heat capacity plays the role of a "thermal mass". The revisited notion of inertia in mechanics will allow to show the analogy between: mechanical inertia (mass), thermal effusivity and electrical inductance. The three parameters show a tendency to keep invariant a certain physical quantity: velocity, temperature and current intensity respectively. However, the analogy is not complete, the capacitance used in the heat transfer seems to be similar to the one used in the Windkessel model which accounts for tube compliance and therefore to a local storage.
\end{abstract}

\section{Introduction}

Building energy efficiency depends on thermal performance of building envelops. Thermal resistance $R$ ( $R$-value) is a good heat transfer parameter for steadystate thermal performance. For transient thermal performance other parameters are needed to capture the time lag and decrement factor, as thermal effusivity $E_{f}$, the so-called thermal mass or the thermal inertia index $D$ [1-4].

The formal electrical analogy is an interesting tool in thermal performance of building envelops studies. In this analogy, the notion of thermal inertia is often related to the capacitance. The electrical analogy is also used in other fields as fluid mechanics. It is surprising that, in the formal electrical analogy, "inertia" is not represented by same component in fluid mechanics and heat transfer. For fluid flow, in the Windkessel model, the fluid inertia is represented by an electrical inductance while in thermal-electric analogy, thermal inertia is given by a capacitance.

Researchers used the terminology "thermal inertia" on the basis of possible similarities with inertia in Mechanics which is the resistance of an object to any change in its state of motion [5]. However other authors argued that « there is no thermal analogy to electrical inductance or "inertia" as [6]. Who think that "Heat capacity" or "thermal capacitance energy storage" terminology used in heat transfer is used incorrectly as "thermal inertia" in the literature. ».

The aim of our communication is to provide some clarification about this controversy. The concept of "inertia" in mechanics will be revisited and we will discuss the concepts of thermal inertia and thermal mass. We will first present the thermal-electric analogy and its application for the prediction of the evolution of indoor temperature. We will discuss then the concept of "Thermal inertia" with the main related parameters such as: thermal effusivity $E_{f}$ and thermal mass. We will present the Windkessel model to show the link with inertia in fluid mechanics.

\section{Thermal-Electrical analogy}

The formal electrical analogy is an interesting tool in thermal performance of building envelops studies. The energy variation in space and time is given by

$\left(\rho C_{p}\right)_{L} \frac{\partial T_{L}}{\partial t}=\lambda_{L} \cdot \nabla\left(\nabla T_{L}\right)$

The index $\mathrm{L}$ is referring to local. Writing (1) on the volume gives

\footnotetext{
* Corresponding author: r.absi@ecam-epmi.com
} 


$$
\begin{aligned}
& \int_{\Omega}\left(\rho C_{p}\right)_{L} \frac{\partial T_{L}}{\partial t} d \Omega=\int_{\Omega} \lambda_{L} \cdot \nabla\left(\nabla T_{L}\right) d \Omega \\
& \text { Or } \\
& \left(\rho C_{p}\right)_{L} \Omega \frac{\partial\left\langle T_{L}\right\rangle}{\partial t}=\int_{\Sigma} \lambda_{L} \cdot \nabla T_{L} \times d \Sigma \\
& \left(\rho C_{p}\right)_{L} \Omega \frac{\partial\left\langle T_{L}\right\rangle}{\partial t}=\int_{\Sigma_{\text {in }}} \lambda_{L} \cdot \nabla T_{L} \times d \Sigma_{\text {in }}+\int_{\Sigma_{\text {out }}} \lambda_{L} \cdot \nabla T_{L} \times d \Sigma_{\text {out }} \\
& \left(\rho C_{p}\right)_{L} \Omega \frac{\partial\left\langle T_{L}\right\rangle}{\partial t}=P(t)+\underbrace{}_{\underbrace{\int_{\text {flux conductif au }} \lambda_{\text {traversmateriaux }} \cdot \nabla T_{L} \times d \Sigma_{\text {out }}}_{\Sigma_{\text {out }}}}
\end{aligned}
$$

And therefore

$$
\frac{d U(t)}{d t}=C_{t h} \frac{d T i(t)}{d t}=P(t)+\Phi(t)
$$

Where $\mathrm{P}(\mathrm{t})$ is referring to heating or cooling or another source term and $\Phi(t)=-\frac{1}{R_{t h}}(T i(t)-T e(t))$; with $T_{e}$ is the outdoor temperature and $T_{i}$ the indoor temperature. Equation (2) represents a capacity of storage.

Finally the equation is given by the following form

$$
C_{t h} \frac{d \operatorname{Ti}(t)}{d t}=P(t)-\frac{1}{R_{t h}}(\operatorname{Ti}(t)-\operatorname{Ta}(t))
$$

The solution is given by

$$
T i(t)=\left(T e(t)+R_{t h} P(t)\right)-\left(T e(t)-T o+R_{t h} P(t)\right) \cdot e^{-t / \tau}
$$

Or

$$
\begin{aligned}
& T i_{P(t) \neq 0}(t)=\left(T e(t)+R_{t h} P(t)\right) \cdot\left(1-e^{-t / \tau}\right)+T o \cdot e^{-t / \tau} \\
& T i_{P(t)=0}(t)=T e(t)+(T o-T e(t)) \cdot e^{-t / \tau}
\end{aligned}
$$

Where, $\tau\left(=R_{t h} \cdot C_{t h}\right)$ is the time constant of the room [sec], $T o$ is the initial temperature $\left[{ }^{\circ} \mathrm{C}\right]$. These equations present the evolution of the indoor temperature of air.

\section{Steady-state case}

$$
\begin{aligned}
& T i(t)=T e(t)+R_{t h} \cdot P(t) \\
& R_{t h}=\frac{T i(t)-T e(t)}{P(t)} \\
& R_{t h} \cong \bar{R}_{t h}=\frac{\overline{T i}-\bar{T} e}{\bar{P}}
\end{aligned}
$$

Where the resistance $R_{t h}$ contains the internal convective resistance $R_{i c}\left[{ }^{\circ} \mathrm{C} / \mathrm{W}\right]$, the resistance of wall $R_{w}\left[{ }^{\circ} \mathrm{C} / \mathrm{W}\right]$ and the external convective resistance $R_{e c}\left[{ }^{\circ} \mathrm{C} / \mathrm{W}\right]$. $R_{t h}=R_{i c}+R_{w}+R_{e c}$

$$
R_{i c}=\frac{1}{h_{i c} A} \quad R_{w}=\sum_{i=1}^{n} \frac{e_{i}}{\lambda_{i} A}, \quad R_{e c}=\frac{1}{h_{e c} A}
$$

\section{Dynamic case}

During the evolution at constant power it is possible to determine the thermal capacity from the known evolution of temperatures.

$$
\begin{gathered}
e^{-t / \tau}=\frac{T i(t)-T e(t)}{T o-T e(t)} \\
\tau=\frac{t}{\ln \left(\frac{T o-T e(t)}{T i(t)-T e(t)}\right)}
\end{gathered}
$$

By using Laplace transform

$$
\begin{aligned}
& C_{t h}(s T i(s)-T i(0))=P(s)-\frac{1}{R_{t h}}(\operatorname{Ti}(s)-T e(s)) \\
& R_{t h} C_{t h}(s T i(s)-T i(0))=R_{t h} P(s)+T e(s)-T i(s) \\
& T i(s)-\frac{T i(0)}{s}=\frac{R_{t h} P(s)+T e(s)-T i(s)}{R_{t h} C_{t h} s}
\end{aligned}
$$

This allows either the simulation via computer tools to predict the evolution of the indoor temperature (figure 1) or in particular cases of outdoor temperature or of power time-variation.

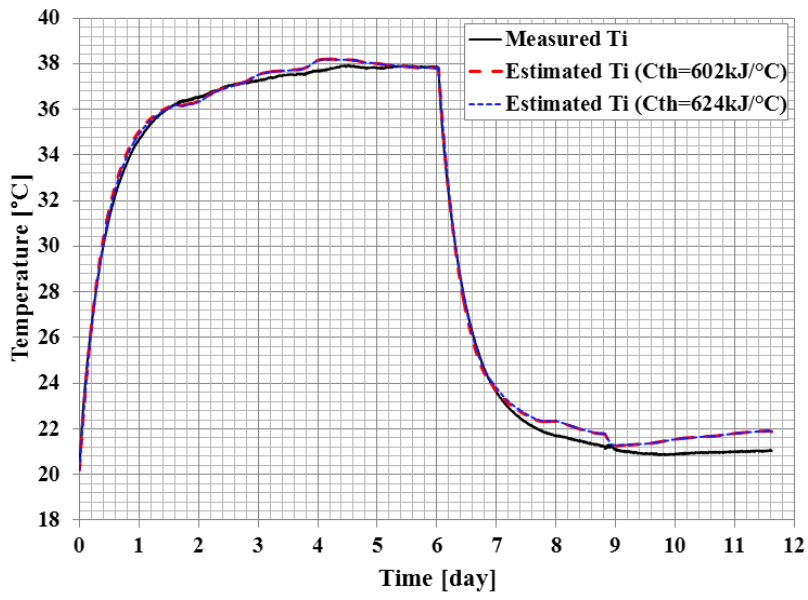

Fig. 1. Simulation with MATLAB for radiative heating (without regulation) and electric convector with setpoint. Analogy with charge-discharge of a capacitor.

The curve, in figure (1), is characteristic of a process which resists the increase in temperature (plateau), before "releasing" this resistance suddenly (here after 6 days), as happens for example during charging or the discharge of a capacitor.

In this analogy the main difference is that the charging and discharging of the capacitor is a discrete phenomenon while the ability to keep or transfer heat is a continuous process. 


\section{Thermal "Inertia"}

Let's first begin by recall the main fundamental notions of thermal conduction.

A material stores a heat $Q$ when its temperature varies by $\Delta T$ in time:

$Q=c m \Delta T$

with : $Q$ in the heat $[Q]=J ; m$ the mass $[\mathrm{m}]=\mathrm{kg} ; \mathrm{c}$ the specific heat capacity $[c]=J k^{-1} K^{-1}$. The volumetric heat capacity is given by $=\rho c ; \rho$ the density in $\mathrm{kg} / \mathrm{m}^{3},[C]=\mathrm{J} \mathrm{m}^{-3} \mathrm{~K}^{-1}$.

In thermal conduction, the heat flux, given by Fourier law, is proportional to the magnitude of the temperature gradient and opposite in sign [7]. The constant of proportionality is the thermal conductivity $\lambda$ or $;[\lambda]=$ $W / m K$. Experimentally, if the temperature variations are not too large, we account locally for the phenomena of heat conduction by the Fourier law.

Or

$$
\overrightarrow{\dot{q}}=-\lambda \overrightarrow{\operatorname{grad} T}
$$

$$
\dot{q}=\frac{\dot{Q}}{S}=\lambda \frac{\Delta T}{L}=\frac{\Delta T}{R}
$$

Where $R=L / \lambda$ is the thermal resistance which is an important parameter in steady-state conduction.

(a)

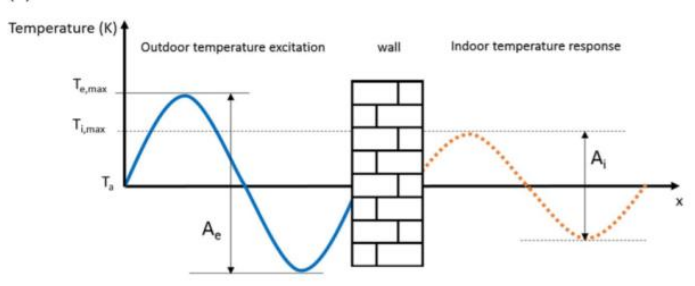

(b)

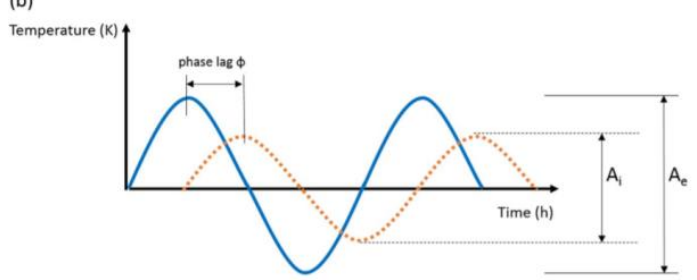

Fig. 2. Thermal response on a sinusoidal heat wave propagating through a wall. Time lag $\phi$ and decrement factor $A_{i} / A_{e}[1]$.

However for transient heat conduction, the phenomenon is presented in figure (2) where the outdoor temperature excitation is compared to indoor temperature response.

The outdoor temperature excitation is given by a sinusoidal-shaped curve given by $A_{e}=T_{e, \max }-$ $T_{e, \min }$. The indoor temperature response has a same shape but shows two main behaviors. The first is a time lag and the second is related to the peak value of temperature which is reduced. The indoor parameter $A_{i}=T_{i, \max }-T_{i, \min }$ is $<A_{e}$, the decrement factor is defined by the ratio $A_{i} / A_{e}$ which is $<1$. This is due to the fact that a given quantity of the heat entering the wall is absorbed and stored inside the materials; the temperature of these materials will increase.

This behavior described by time lag $\phi$ and decrement factor $A_{i} / A_{e}$ depends on the thermo-physical properties of the construction materials.

The involved parameters are the mass of the materials, the thermophysical properties and the thickness of walls [8-9].

The capacity to accumulate heat is therefore a property of a material. It is linked to both thermal conductivity $\lambda$ and volumetric heat capacity $C=\rho c$. We have therefore a phenomenon which results from two distinct contributions namely the volumetric heat capacity and thermal conductivity.

It is interesting to define a unique parameter to evaluate and quantify the capacity of a body/material to accumulate heat (heat storage). The idea is to merge these two contributions within a unique parameter. It is more judicious to call it the capacity of heat storage $C_{h s}$ (or capacity to accumulate heat). The entire question is how to define this unique parameter which merges these two contributions.

However, the thermal diffusivity $\alpha$ is defined from these two parameters. The one-dimensional heat diffusion equation allows to find the temperature distribution $T(x, y)$. The heat diffusion equation includes the thermal diffusivity $\alpha$ which is the ratio between thermal conductivity $k$ and volumetric heat capacity $C=\rho c$.

$\alpha=\frac{\lambda}{C}=\frac{\lambda}{\rho c}$

Where $[\alpha]=m^{2} / s$. Thermal diffusivity is a parameter which contains both contributions related to thermal conductivity $\lambda$ and volumetric heat capacity $C$. Why the thermal diffusivity is not very used in practice? and why the need to define other parameters?

Equation (6) shows that diffusivity represents the relationship between two trends: transport and heat accumulation. It is a certain measure of the "competition" between the capacity of the material to transport heat, and its capacity, at the opposite, to store it. However, thermal conductivity $\lambda$, as the thermal capacity $C$, does not have concurrent effects when we are interested - which is our case - about the notion of thermal inertia. In this case, these two parameters come to cumulate their effects, to limit the temperature differences and best ensure its regulation. Thermal conductivity achieves this by promoting the transport of heat, which tends to reduce the spatial temperature gradient and therefore to produce a uniform temperature 
at any point in the material. The thermal capacity limits the temperature variations of the material over time, thus tending to ensure a temporal invariance of the temperature. In a certain sense, thermal conductivity marks the tendency towards a certain homogeneity of temperature; thermal capacity a tendency towards a certain stationarity. These two parameters combine their effects towards the thermal inertia concept of matter: tendency to keep its temperature invariant (in space as in time).

Another method is to use the geometric mean which is a mean or average, which indicates the central tendency by using the product of their values, as opposed to the arithmetic mean which uses their sum. The use of the geometric mean, which is the square root of the product of thermal conductivity and volumetric heat capacity, allow to define the thermal effusivity given by

$E_{f}=\sqrt{\lambda C}$

Where: $\lambda$ is the thermal conductivity in $W / m K$ and $C$ the volumetric heat capacity, $[C]=J m^{-3} K^{-1}$. The unit of $E_{f}$ is therefore in $J / m^{2} K s^{0.5}$ Thermal effusivity is a geometric mean of the two contributions: The proper heat of the material or static heat given by Eq. (4) and the heat evacuated or transferred (transported) given, in conduction, by Fourier's law given by Eq. (5).

It is therefore not the thermal diffusivity $\alpha$ which can measure the thermal inertia of matter, but on the contrary the thermal effusivity defined by equation (7) which, as we can see, does not put into competition $\lambda$ and $\mathrm{C}$ (unlike $\alpha$ ), but cumulatively takes into account the inertial properties of these two parameters, of which it is the geometric mean.

The Chinese thermal design code for civil building (GB 50176 - 201X) [10], the thermal inertia index $D$ characterizes the transient thermal performance. It is given by

$D=R S$

where $R$ is the thermal resistance, $m^{2} K / W ; S=$ $\sqrt{(2 \pi \lambda C) / T}$ is the coefficient of thermal storage, $W / m^{2} K$. It is a dimensionless index reflecting the counteraction of exterior wall on fluctuations in temperature and heat flow. It is possible to write a link between $D$ and $E_{f}$ as

$$
D=(L / \lambda) \sqrt{(2 \pi) / T} E_{f}
$$

Which seems related to the Entransy [11].

\section{Inertia in fluid mechanics}

In fluid mechanics inertia is associated to the mass of the fluid. In hemodynamic, when using the wellknown Windkessel model for blood flow, the fluid inertia is represented by an electrical inductance. The block diagram is described by two signals: the inlet flow and the outlet pressure difference. This indicates that a pressure variation is linked to a decrease/increase in the flow rate.

A thermal/fluid flow analogy could be described by block diagram. In thermal applications, the block diagram is given by two signals: the heat flow at the inlet and the temperature difference at the outlet. This indicates that a change in temperature is linked to heat gain/loss.

The Windkessel model, which allows adequate quantification of pressure changes during the systolic and diastolic phases of the cardiac cycle, is based on an electrical analogy. It provides time-dependent pressure during the systolic and diastolic phases [12-13]. In the 2Element Windkessel Model, the resistance of the arterial system and arterial compliance are represented respectively by a resistor and a capacitor (fig. 3.a). In the 4- Element Windkessel Model, in addition to an added resistor which account for this resistance to blood flow due to the aortic valve, an inductor is included to account for the inertia of blood flow in the hydrodynamic model (fig. 3.b).

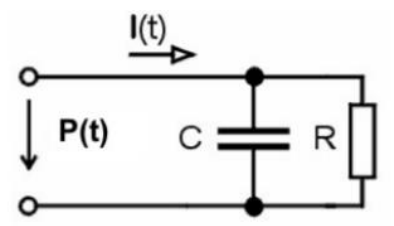

(a)

Electrical Analog of the 2-Element Windkessel Model

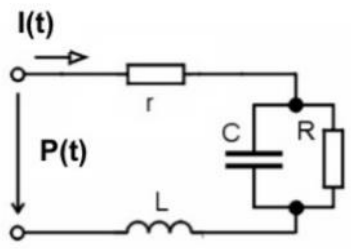

(b) Electrical Analog of the 4-Element Windkessel Model

Fig. 3. Flow-electrical analogy; (a) 2-Element model, (b) 4Element model; with inertia: inductance L.

The Windkessel model is validated by experimental data. Blood flow information was acquired using a cardiacgated, 2D, respiratory compensated, phase-contrast (PC) cine sequence with through-plane velocity encoding [14]. The cardiac output of the patient was $3.71 \mathrm{~L} / \mathrm{min}$, the heart rate 47 beats per minute (cardiac cycle $\mathrm{T}=$ $1.277 \mathrm{sec}$ ). Figure (4) shows measured and reconstructed time-dependent flow rate $Q_{t}$ at the level of the ascending aorta. A 15-term Fourier reconstruction of the flow waveforms is given by [14] 


$$
Q_{t}=\operatorname{real}\left\{\sum_{n=0}^{14} Q_{n} e^{i \omega n t}\right\}
$$

where $Q_{n}$ is the Fourier mode and $i$ is the imaginary unit.

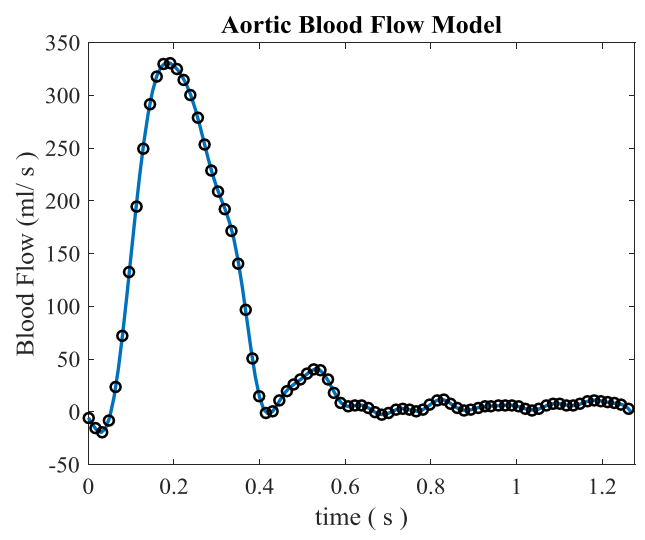

Fig. 4. Pulsatile blood flow. Ascending aortic flow waveforms (in $\mathrm{ml} / \mathrm{sec}$ ) measured by the (PC)-MRI sequence [14].

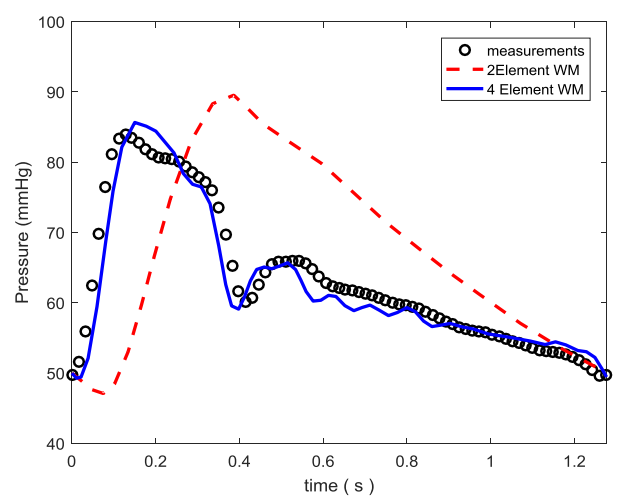

Fig. 5. Pressure. Dashed-line (--): 2-Element model; Solid line: 4-Element model (with inertia: inductance L).

Figure (5) shows time-dependent pressure during the systolic and diastolic phases obtained from the Windkessel model. The dashed line represents the result of the 2-Element Windkessel Model while the solid line is the result of the 4- Element Windkessel Model which allows an accurate description of the measured values (symbols). This is because the 4- Element Windkessel Model accounts all effects: resistances/resistors, arterial compliance/capacitor and especially inertia of blood flow/inductor.

For the 2-Element model (without inductance, dashed line), the peak value of pressure shows an important shift (or phase-lag). The 4-Element model allows removing this shortcoming thanks to the inductance which takes into account the fluid mass or fluid inertia. Both model and experimental peak values are located at same time.

The electrical analogy in fluid mechanics, where inertia is represented by an inductor, could be explained by the magnetic field flux of the inductance. Since in electricity, the inductance increases the magnetic field flux creating an induced current which decreases the main current. This phenomenon is similar to the inertia due to bodies' motion and flowing fluids.

\section{Discussion and concluding remarques}

Revisiting the notion of inertia in mechanics is important to better understand the analogy.

In mechanics, Inertia is related to the motion of a physical object. It is the resistance of an object to any change in its state of motion. From Newton's law it is easy to find a relation between the mass of a solid and its acceleration. The variation of velocity is inversely proportional to the Mass ("Inertia"). This relation is given by

$\Delta v \approx \frac{1}{M}$

Where $\Delta v$ is the velocity variation and $M$ is the mass. This is obvious, since heaviest is the mass, less easy its acceleration becomes.

In mechanics, Equation (10) provides a relation between the mass $M$ and the variation of velocity $\Delta v$. It is a good tool to explain, by analogy, the notion of "thermal mass". The temperature difference $\Delta T$ is inversely proportional to thermal effusivity $E_{f}$ since $\Delta T$ is inversely proportional to $C$ on the one hand and to $\lambda$ on the other, and therefore

$\Delta T \approx \frac{1}{E_{f}}$

Comparison between (10) and (11) shows that thermal effusivity plays the role of a "thermal" mass. Mechanical inertia, i.e. the inertia of motion, of which the fundamental measure is the mass $\mathrm{M}$ of bodies, tends to regulate their velocity (by limiting their accelerations), as thermal inertia regulates their temperature. This confirms that the thermal effusivity $E_{f}$ in heat transfer plays the role of the mass $\mathrm{M}$ in mechanics.

In the electrical analogy, impedance represents the inertia. Since, the mass regulates the velocity by limiting the production of accelerations, as the impedance regulates the current (or the velocity of charges) by limiting the potential differences in a conductor. Therefore, mass and impedance play symmetrical inertial roles.

All of the above clearly highlights the analogy between: mechanical inertia (mass), thermal inertia (effusivity: more general than thermal capacity in the presence of transport mechanisms) and electrical inertia (inductance). The three parameters show a tendency to keep invariant a certain physical quantity: velocity, temperature and current intensity respectively. 
Revisiting the notion of inertia in mechanics has therefore allowed us to clearly visualize the analogy between mass and effusivity, hence the name "thermal mass" given to effusivity. The thermal effusivity which is the geometric mean of thermal conductivity and volumetric heat capacity represents therefore the thermal mass.

However, the formal electrical analogy seems not complete between fluid-flow and thermal phenomena. In the Windkessel model for fluid flow in elastic tubes, "inertia" is related to the electrical inductance while the capacitor is used to describe the compliance of elastic tube walls which allow a certain volume of fluid to be stored during the flow. However, in thermal performance of building envelops, capacitance is used to describe the storage and release of heat as a charge and discharge of a capacitor. The capacitance used in the thermal-electric analogy seems to be similar to the one used in the Windkessel model which accounts for tube compliance and therefore to a local storage. In this case, the terminology of "thermal inertia" is used incorrectly as argued by Shah and Sekulic [6]. Thermal effusivity is therefore a parameter related to the capacity of heat storage (capacity of a body/material to accumulate heat) and thermal mass but not to "inertia" in this particular sense.

\section{References}

1. S. Verbeke, A. Audenaert, Renewable and Sustainable Energy Reviews 82, 2300 (2018).

2. H. Asan, Y.S. Sancaktar, Energy Buildings 28, 159 (1998).

3. H. Asan, Energy Buildings 32 (2) 197 (2000).

4. R. Cheng, X. Wang, Y. Zhang, Energy and Buildings 80598 (2014).

5. C. A. Balaras, Overv Comput Methods Energy Build 24, 1 (1996).

6. R. K. Shah, D. P. Sekulic, 2003, John Wiley \& Sons, 976 pages.

7. Y. A. Cengel, Heat and Mass Transfer: A Practical Approach. Third Edition. McGrawHill

8. K. Ulgen, Energy Build 34, 273 (2002).

9. P. Tuohy, L. McElroy, C. Johnstone, In: Proceedings of the ninth int IBPSA conf; 1253-60 (2005).

10. China Academy of Building Research, Thermal Design Code for Civil Building (GB 50176-201 X), China Building Industry Press, Beijing, 2014 (in Chinese).

11. Z.-Y. Guo, H.-Y. Zhu, X.-G. Liang, International Journal of Heat and Mass Transfer 50, 2545 (2007).

12. O. Frank, The basic shape of the arterial pulse. First treatise: Mathematical analysis Translation of Otto frank's paper "Die Grundform des arteriellen Pulses”, Zeitschrift Für Biologie, 37, 483 (1899).
13. D. Guan, F. Liang, P. A. Gremaud, Journal of Biomechanics, 49(9), 1583 (2016).

14. C. Karmonik, A. Brown, K. Debus, J. Bismuth, A. B. Lumsden, CFD Challenge: Predicting PatientSpecific Hemodynamics at Rest and Stress through an Aortic Coarctation. STACOM 2013. Lecture Notes in Computer Science, vol 8330. Springer, Berlin, Heidelberg (2014). 